\title{
EDITORIAL
}

\section{Immune systems in skin wound healing}

\author{
Toshikazu Kondo \\ Department of Forensic Medicine, Wakayama Medical University, Japan \\ CORRESPONDING AUTHOR \\ Toshikazu Kondo. Department of Forensic Medicine, Wakayama Medical University, Japan; kondot@wakayama-med.ac.jp \\ CITATION \\ Kondo T. Immune systems in skin wound healing. Trends Immunother 2017; 1(1): 57. doi: 10.24294/ti.v1.i2.106.
}

Tissue repair in every organ is one of the most important biological phenomena. Skin is located outside of the body, and is always exposed to various kinds of external stimulation such as radiological, thermal and mechanical stimulation. Thus, skin plays essential roles in the protection from outer stimulation as the barrier. After disruption of skin structure due to various assaults, the damaged skin structure would be repaired in accordance with the passage of time, which is called skin wound healing ${ }^{[1]}$. This healing process immediately starts after injury and is composed of three phases: inflammation, proliferation, and maturation. These phases proceed with complicated but well-organized interaction between extracellular matrices and epithelia, endothelia and immune cells. Moreover, lots of cellular and molecular biological studies demonstrated that many cytokines, growth factors, and proteases are closely involved in the wound healing process to complete normal tissue repair after damage.

We are focusing on the molecular mechanism of skin wound healing. Since development of genetic engineering in animals, molecular mechanical insights of skin wound healing have been clarified using knockout or transgenic mice. Our group also carried out a systemic research project of skin wound healing using knockout mice. In particular, we have much interest in the pathophysiological roles of inflammatory mediators such as cytokines and chemokines in skin wound healing. It is wellknown that (interleukin-1) IL-1, IL-6 and (tumor necrosis factor $\alpha$ ) TNF $\alpha$ are essential and classical inflammatory cytokines, and they are therapeutic molecular targets in inflammatory diseases such as rheumatoid arthritis, psoriasis and inflammatory bowel disease. Our research implied an essential involvement of IL-6 in the skin wound-healing process as shown by reduced immune cell recruitment and angiogenesis, and eventual impaired wound healing in IL-6-deficient mice ${ }^{[2]}$. The genetic disruption of IL-1 receptor antagonist (IL-1 ra) in mice could enhance IL-1 bioactivity, resulting in the exaggeration of inflammatory responses after wounding ${ }^{[3]}$. Actually, the absence of IL-1ra delayed wound healing with exacerbated local inflammatory. On the contrary, the absence of TNF-signaling in the disruption of TNF receptor p55 reduced in- flammatory reactions but promoted angiogenesis and collagen production, eventually accelerating skin wound healing ${ }^{[4]}$. Collectively, excessive inflammatory responses had detrimental roles in skin wound healing.

During skin wound healing, (transforming growth factor- $\beta$ ) TGF- $\beta$ signals can promote collagen production in the proliferative phase. On the contrary, interferon- $\gamma$ (IFN- $\gamma$ ) suppresses collagen production in fibroblasts through attenuation of TGF- $\beta$ signals, implying that signal cross-talk is essential in skin wound healing ${ }^{[5]}$.

Finally, skin wound healing is well-regulated immune responses. The manipulation of these immune responses could have influence on the event of skin wound healing. Thus, it is necessary to explore key molecules in skin wound healing, eventually resulting in the development of immunotherapy for skin wound healing.

\section{References}

1. Kondo T, Ishida Y. Molecular pathology of wound healing. Forensic Sci Int 2010; 203(1-3): 93-98. doi: 10.1016/j.forsciint.2010.07.004.

2. Lin ZQ, Kondo T, Ishida $\mathrm{Y}$, et al. Essential involvement of IL-6 in the skin wound-healing process as evidenced by delayed wound healing in IL-6deficient mice. J Leukoc Biol 2003; 73(6): 713721. doi: $10.1189 / \mathrm{jlb} .0802397$.

3. Ishida Y, Kondo T, Kimura A, et al. Absence of IL-1 receptor antagonist impaired wound healing along with aberrant NF- $\kappa B$ activation and a reciprocal suppression of TGF- $\beta$ signal pathway. J Immunol 2006; 176(9): 5598-5606. doi: 10.4049/ jimmunol.176.9.5598.

4. Mori R, Kondo T, Ohshima T, et al. Accelerated wound healing in tumor necrosis factor receptor p55-deficient mice with reduced leukocyte infiltration. FASEB J 2002; 16(9): 963-974. doi: 10.1096/fj.01-0776com.

5. Ishida $\mathrm{Y}$, Kondo T, Takayasu $\mathrm{T}$, et al. The essential involvement of cross-talk between IFN- $\gamma$ and TGF- $\beta$ in the skin wound-healing process. J Immunol 2004; 172(3): 1848-1855. doi: 10.4049/ jimmunol.172.3.1848. 